\title{
Cyclosporin in the treatment of severe chronic idiopathic uveitis
} J de Vries, G S Baarsma, M J W Zaal, T N Boen-Tan, A Rothova, H J Buitenhuis,
C M C Schweitzer, R J W de Keizer, A Kijlstra cyclosporin. Preliminary data like the effects on second set rejection of allografts in animal $^{\mathrm{s}-7}$ and man, ${ }^{8}$ the results in the treatment of graft-versushost disease in man, ${ }^{9}$ and the effects in experimental animals of uveitis ${ }^{10-13}$ imply its possible usefulness.

In 1983 Nussenblatt and associates ${ }^{14}$ published the first study in which cyclosporin was used as a treatment of intraocular inflammatory disease in man. They concluded that 'cyclosporin A appears to be an effective alternative to the present therapies'. Since then similar results have been found in other case reports and pilot studies. ${ }^{15-21}$

Until now no double-masked placebocontrolled studies have been published in which these first impressions have been either rejected or confirmed.

\section{Patients and methods}

ORGANISATION

Four ophthalmological departments participated in this study. The Rotterdam Eye Hospital (the Department of Ophthalmology of Erasmus University) was responsible for collecting and analysing the data. The Netherlands Ophthalmic Research Institute provided communications. infection such as Toxoplasma gondii, toxocara, candida, Mycobacterium tuberculosis, Treponema pallidum, or herpes viruses can be established. ${ }^{1}$ The other uveitis cases are 'idiopathic', though in most of the patients a clinical entity like Behçet's disease, sarcoidosis, or birdshot retinochoroidopathy can be diagnosed.

For these cases of 'idiopathic' uveitis corticosteroids are the mainstay of therapy. In severe chronic idiopathic uveitis chemotherapeutic agents such as chlorambucil and azathioprine are the only recourse if corticosteroids are ineffective or not tolerated. Most physicians are reluctant to use chemotherapeutic agents, however, because of potential toxicity including neoplasia, bone marrow depression, secondary opportunistic infection, and sterility. The introduction of cyclosporin, a new immunosuppressive agent, offered the hope of a safer and more effective therapy for patients with an intractable, blinding uveitis.

Experience in organ transplantation proved that cyclosporin can effectively prevent the initiation of an immune response to foreign antigens. ${ }^{2-4}$ In idiopathic uveitis, however, the situation is completely different. Here the immune response is already in full swing with (auto)antigens recognised, helper cells activated, and effector clones established. Little is known about the therapeutic instead of preventive use of

\section{STUDY DESIGN}

The investigation was designed as a randomised, double-masked, placebo-controlled clinical trial. The factors analysed were the efficacy, safety, and tolerability of cyclosporin.

After obtaining informed consent each patient was allocated in a strictly consecutive order to either the cyclosporin group or the placebo group according to a randomisation list. After inclusion in the study the patients were reexamined at week 1, 2, and 4 and at one-month intervals thereafter for up to one year.

All patients were monitored by the same two investigators throughout the study. One masked investigator, with no information about the assigned treatment, evaluated the efficacy parameters and decided about possible changes in therapy according to the protocol. A second investigator who knew the assigned treatment checked the safety parameters and recorded the subjective side effects.

\section{ELIGIBILITY CRITERIA}

Patients were eligible for admission to the study if they had an active idiopathic posterior uveitis, panuveitis or intermediate uveitis, an insuffici- 
ent response to systemic corticosteroids, and a best corrected visual acuity of 0.5 or less in their best eye. This last criterion did not apply for patients with Behçet's disease or sympathetic ophthalmia, since they can be well separated from the other uveitis cases and since their poor prognosis under current therapy is well documented. ${ }^{22} 23$

The following exclusion criteria were applied: age under 18, presumed infectious uveitis, endstage disease with irreversible retinal damage, corneal or lens opacities preventing the evaluation of the efficacy parameters, anticipated intraocular surgery during the study, contraindications to immunosuppression (that is, uncontrollable infections, malignancy, or a history of malignancy), contraindications to oral corticosteroid therapy, concomitant therapy with cytostatic agents or nephrotoxic medicines, impaired kidney or liver function, hypertension, pregnancy, malabsorption syndrome, drug or alcohol abuse, and non-cooperation.

TREATMENT PLAN

At entry into the study all patients received a low dose of prednisone $(0.3 \mathrm{mg} / \mathrm{kg} /$ day to a maximum of $20 \mathrm{mg} /$ day). In one group this was combined with cyclosporin in a single dose of 10 $\mathrm{mg} / \mathrm{kg} /$ day, while the second group received a placebo instead. After two weeks of therapy and depending on the clinical response (see below) the corticosteroid dosage was reduced by $2.5 \mathrm{mg}$ per fortnight and eventually stopped. After oral corticosteroids had been tapered off to zero, cyclosporin (or placebo) was reduced by $1 \mathrm{ml}$ $(=100 \mathrm{mg})$ per month. In Figure 1 an example is given of the dose reduction protocol for a $70 \mathrm{~kg}$ patient, in case of treatment success. The dosages were tapered off in accordance with protocol in order to compare the two treatment groups with regard to the number of months of successful therapy before the uveitis relapsed. The dosage of corticosteroids or cyclosporin (or placebo) could be kept constant only in case of a decrease in visual acuity of 1 rank number (see efficacy parameters), compared with the best visual acuity, owing to uveitis, or in case of an unchanged visual acuity in combination with an increase in inflammatory activity score of more than 4 points.

For safety reasons the dose of cyclosporin drink solution could be reduced by $25 \%$ in case of a predose ( 24 hours after the last dose) cyclosporin concentration in whole blood

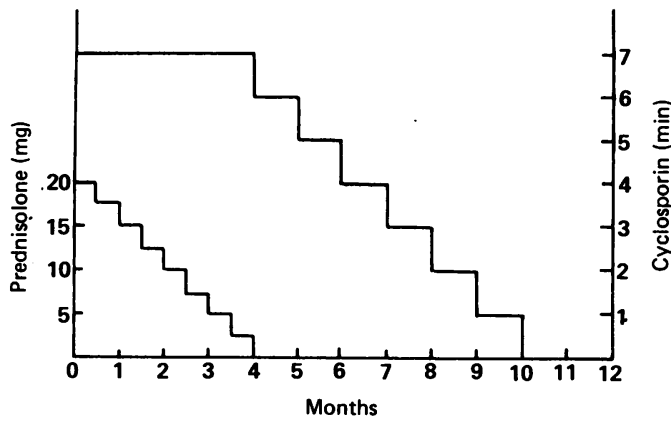

Figure 1: Example of the dose reduction protocol for a $70 \mathrm{~kg}$ patient in case of treatment success. exceeding $1000 \mathrm{ng} / \mathrm{ml}$, an increase of serum creatinine level above $150 \%$ of the baseline value, an increase of the liver function parameters above $200 \%$ of the upper normal limits, or hypertension (diastolic blood pressure above 95 $\mathrm{mmHg}$ for age $\leqslant 50$ or above $100 \mathrm{mmHg}$ for age $>50$ ). In order to safeguard the double-masked design of the study dose reductions were also made in the placebo group. The dosage of corticosteroids or cyclosporin (or placebo) could not be increased. The only concomitant medication for uveitis allowed was dexmethasone $0 \cdot 1 \%$ and/or atropine $1 \%$ eye drops. Subconjunctival or parabulbar injections of corticosteroids were not allowed.

A combination therapy was given because a double-masked comparison of cyclosporin versus prednisone was not possible owing to the different administration forms and the outward side effects of corticosteroids. Prednisone was given in a low dose to avoid overimmunosuppression and the resulting increased susceptibility to malignant neoplasms and infections.

\section{EFFICACY PARAMETERS}

The main efficacy parameter was the visual acuity. The best corrected visual acuity was determined at $6 \mathrm{~m}$ with charts which contain Landolt $\mathrm{C}$ optotypes ranging in unequal steps from a visual angle of $10^{\prime}$ (that is visual acuity $20 /$ 200 ) to one of $0.5^{\prime}$ (visual acuity 20/10). When the visual acuity of a patient was below $20 / 200$ a second ordinal scale was used, namely, finger counting (FC), hand movements (HM), light perception (LP), and no light perception (NLP).

In order to make comparisons between the two measurement scales the visual acuity of each eye was given a rank number. For example, visual acuities of hand movements in one eye and 20/80 in the other were given the rank numbers 2 and 8 respectively.

The second efficacy parameter was the inflammatory activity. This was assessed by using a slightly modified Hogan-Thygeson-Kimura scale. ${ }^{24}$ This scale is shown in Table $\mathrm{I}$. The inflammatory activity score was the sum of the scores of the individual scores. Owing to the large variety of signs and symptoms included in the scale, a maximal score of 52 could theoretically be reached. In the clinical setting, however, this maximal score could not be reached because the severity of one parameter made impossible

TABLE I Inflammatory activity score

\begin{tabular}{|c|c|c|c|c|c|}
\hline \multirow{2}{*}{$\frac{\text { Parameter }}{\text { Congestion }}$} & \multicolumn{5}{|c|}{ Score } \\
\hline & $0^{\star}$ & & 2 & & $4 \dagger$ \\
\hline Keratic precipitates & 0 & & 2 & & 4 \\
\hline Anterior chamber flare & 0 & 1 & 2 & 3 & 4 \\
\hline Anterior chamber cells & 0 & 1 & 2 & 3 & 4 \\
\hline Vitreous opacity & 0 & 1 & 2 & 3 & 4 \\
\hline Macular oedema & 0 & & 2 & & 4 \\
\hline Optic disc oedema & 0 & & 2 & & 4 \\
\hline Vasculitis & 0 & & 2 & & 4 \\
\hline Infiltrates & 0 & & 2 & & 4 \\
\hline Snowballs & 0 & & & & 4 \\
\hline Snow banks & 0 & & & & 4 \\
\hline Exudates & 0 . & & & & 4 \\
\hline Haemorrhages & 0 & & & & 4 \\
\hline
\end{tabular}

^Absent; †Strongly present. 
the evaluation of other more posteriorly situated parameters, that is, vitreous opacity versus macular oedema.

\section{SAFETY PARAMETERS}

The following safety parameters were recorded: cyclosporin blood level, serum creatinine, urea nitrogen, serum aspartate transaminase (SGOT), serum alanine transaminase (SGPT), bilirubin, $\gamma$-glutamyl transpeptidase $(\gamma$-GT), lactic dehydrogenase (LDH), haemoglobin, white blood cell and differential counts, platelets, erythrocyte sedimentation rate, blood pressure, pulse rate, and body weight in standardised conditions. Cyclosporin blood levels were measured by using the radioimmunoassay kit manufactured by Sandoz (Basle, Switzerland). Cyclosporin was taken orally once daily at breakfast. Patients were instructed to postpone taking their medication on the morning of re-examination to allow determination of the predose cyclosporin blood level.

\section{END POINTS}

For the individual patient the study lasted one year. A premature termination was considered as either 'treatment failure' or 'drop out'. A treatment failure was defined as $(a)$ a decrease in visual acuity of $\geqslant 2$ rank numbers compared with the best visual acuity, due to uveitis, or $(b)$ an unchanged visual acuity in combination with an increase in inflammatory activity score of $\geqslant 4$ points, or $(c)$ a discontinuation of the medication because of side effects which did not respond to dose reduction. A patient was considered a dropout in case of withdrawal of consent, noncompliance, intraocular surgery, occurrence of contraindications to immunosuppression, pregnancy, or when lost to follow-up

\section{STATISTICS}

The findings from all the patients who entered the study were analysed. To account for those patients who dropped out during the study the efficacy results were expressed in Kaplan-Meier estimates of the 'survival' curves for the two treatment groups.

The two-sided Wilcoxon's rank sum test was used for quantitative variables and the log rank

TABLE II Comparison of treatment groups

\begin{tabular}{lll}
\hline Characteristic & Cyclosporin & Placebo \\
\hline Number of patients & 14 & 13 \\
Male/female ratio & $6 / 8$ & $7 / 6$ \\
Age (yr) & $44 \cdot 5(19 \cdot 5,22-74)^{\star}$ & $45 \cdot 6(15 \cdot 9,26 \cdot 75)$ \\
Visual acuity & $20 / 125(\mathrm{FC}, 20 / 64) \dagger$ & $20 / 125(\mathrm{FC}, 20 / 80)$ \\
Inflammatory activity & $12 \cdot 3(6 \cdot 2,3-24)$ & $11 \cdot 9(4 \cdot 1,5-20)$ \\
Duration of disease (yr) & $5 \cdot 6(4 \cdot 2,1-16)$ & $6 \cdot 5(6 \cdot 2,1-26)$ \\
Body weight (kg) & $67 \cdot 5(9 \cdot 5,53-86)$ & $67 \cdot 8(9 \cdot 6,52-86)$ \\
Diagnostic subgroups: & 3 & 1 \\
Behçet's disease & 4 & 5 \\
Intermediate uveitis & - & 1 \\
Birdshot retinochoroidopathy & 2 & 1 \\
Sarcoidosis & -3 & 2 \\
Vasculitis & 2 & 2 \\
Panuveitis & & \\
Chorioretinitis & & \\
\hline
\end{tabular}

* Mean with SD and range in parenthesis. None of the differences between the groups was statistically significant at the $5 \%$ level; $+\mathrm{FC}=$ finger counting. test for the comparison of the 'survival' curves.

The study protocol was approved of by the ethical committees of the participating university hospitals.

\section{Results}

Twenty-seven patients with a chronic idiopathic posterior uveitis, panuveitis, or intermediate uveitis entered the study. All patients had been unsuccesfully treated with high doses of systemic corticosteroids previously. Two patients had also recieved cytostatic agents on previous occasions. In all cases an active intraocular inflammation of non-infectious origin was present at entry. Fourteen patients were randomly allocated to cyclosporin and 13 to placebo. Table II summarises the background characteristics of the patients.
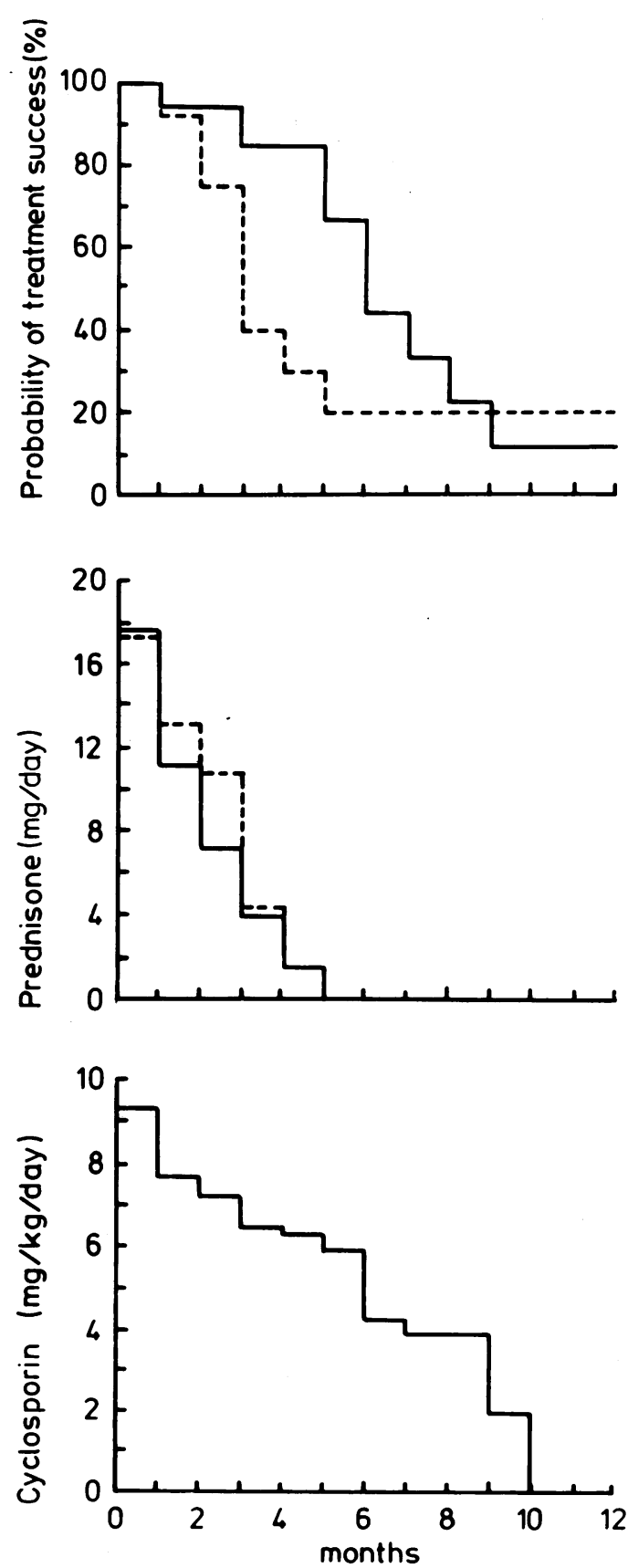

Figure 2: Probability of treatment success, mean prednisone dose, and mean cyclosporin dose in 27 patients with severe chronic idiopathic uveitis, given either prednisone and cyclosporin (continuous line) or prednisone and placebo (dotted line). 


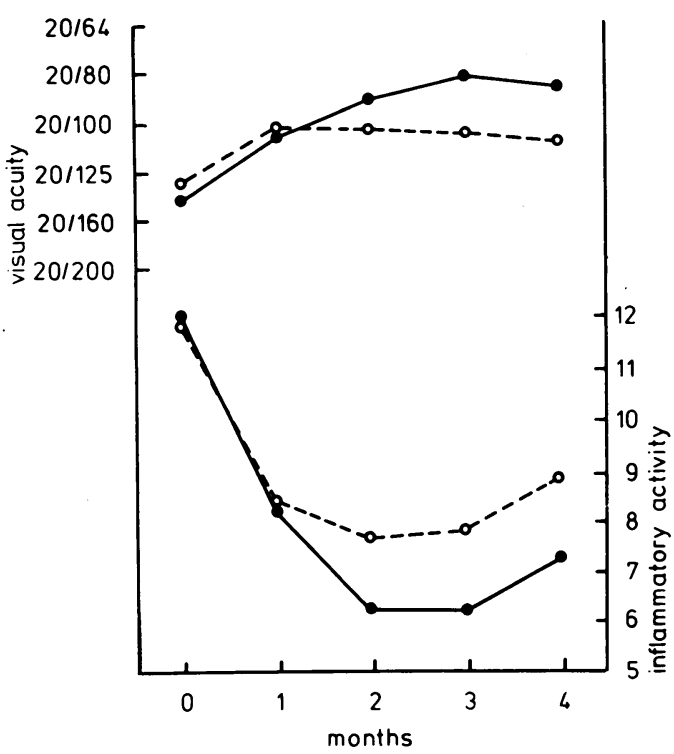

Figure 3: Course of the mean visual acuity and mean inflammatory activity in 27 patients with severe idiopathic uveitis, given either prednisone and cyclosporin (continuous line) or prednisone and placebo (dotted line). p>0.05. Wilcoxon's rank sum test, two sided.

None of the differences between the groups was statistically significant at the $5 \%$ level. Only one patient of the cyclosporin group had a visual acuity of more than 0.5 in his best eye at entry. This patient had Behçet's disease.

\section{EFFICACY}

The efficacy results are presented in Figure 2. The cyclosprin group fared better than the placebo group in number of months of successful therapy. However, the positive effect of cyclosporin was not lasting after dose reduction, and the difference between the two 'survival' curves did not reach statistical significance $(p=0.155$, log rank test).

The cyclosporin concentration in blood at the moment of withdrawal from the study because of treatment failure ranged from 0 to $781 \mu \mathrm{g} / \mathrm{l}$, with a mean at $286 \mu \mathrm{g} / \mathrm{l}$. All nine patients of placebo group still used prednisone (mean $12 \cdot 2$, range $5-20 \mathrm{mg} /$ day) at that moment, whereas for only three patients of the cyclosporin group this was still the case $(5,10$, and $20 \mathrm{mg} /$ day, respectively).

As shown in Figure 3, the course of the mean visual acuity and inflammatory activity appeared to be the same for both treatment groups. A synergistic immunosuppressive effect of the combination of prednisone and cyclosporin could not be demonstrated.

In all except two patients treatment failure was due to a decrease in visual acuity of 2 lines or more in comparison with the best visual acuity.

TABLE III Incidence of abnormal safety parameters during therapy of idiopathic uveitis with cyclosporin

\begin{tabular}{lll}
\hline & \multicolumn{2}{l}{ Number of patients } \\
\cline { 2 - 3 } Safety parameter & Cyclosporin & Placebo \\
\hline Creatinin clearance reduction $>25 \%$ & 4 & 1 \\
Proteinuria & 4 & 1 \\
Bodyweight increase $>5 \mathrm{~kg}$ & 5 & 3 \\
Diastolic blood pressure increase $>15 \mathrm{mmHg}$ & 4 & 0 \\
\hline
\end{tabular}

The other two patients (one in each treatment group) were withdrawn because of a decrease in visual acuity of 1 rank number in combination with an increase in inflammatory activity score of more than 4 points. Medication never had to be discontinued because of side effects, though in some cases a dose reduction was necessary because the trough cyclosporin blood levels exceeded $1000 \mathrm{mg} / \mathrm{l}$. Only one patient in each group was considered a treatment success during 12 consecutive months. At termination of the study one patient of the cyclosporin group had not yet completed the follow-up period of 12 months.

During the study there were three drop-outs in each treatment group. In the cyclosporin group one patient moved abroad and was lost for follow-up, one did not comply with the protocol, and one developed cataract which made evaluation of the efficacy parameters impossible. In the placebo group one patient had to undergo glaucoma surgery, one developed cataract, and one had unexplained positive cyclosporin blood levels, probably owing to an accidental change in assigned medication. The possibility of false positive cyclosporin blood levels as put forward by Johnston et $a l^{25}$ was rejected because the patient had positive cyclosporin levels on two consecutive occasions and a simultaneous rise in serum creatinine level. Although the particular placebo sample could not be analysed, other samples of the same batch were negative for cyclosporin. The patient was classified as a 'drop-out' at month 6.

\section{SAFETY}

Renal function was assessed by serum creatinine concentrations. Under treatment three patients of the cyclosporin group had an increase in serum creatinine concentration exceeding 50\% of the baseline value; one patient for three consecutive months and the others for only one month. Interestingly also one patient of the placebo group had such an increase for one month. This patient was diagnosed as having an intermediate uveitis. No patient received other drugs known to be nephrotoxic. An estimation of the creatinine clearance was made by using the formula of Cockcroft and Gault. ${ }^{26}$ Only one patient of the placebo group had a reduction in creatinine clearance exceeding $25 \%$ of the baseline value for one month, while four patients in the cyclosporin group had such a reduction (Table III). A trace of proteinuria was occasionally found in three patients of the placebo group and four patients of the cyclosporin group. Only one of these patients also had a reduction in renal function creatinine clearance) during the trial.

All liver function values were normal at the start and only showed minor fluctuations within the normal range during treatment.

The haematological parameters showed very slight fluctuations above or below the normal range during the study. No change in erythrocyte sedimentation rate was noticed, either in the cyclosporin group or in the placebo group.

All patients had a normal diastolic blood pressure at entry (that is, $\leqslant 95 \mathrm{mmHg}$ ). This remained so for all patients of the placebo group, 
TABLE IV Side effects as reported by the patients

\begin{tabular}{lll}
\hline & \multicolumn{2}{l}{ Number of patients } \\
\cline { 2 - 3 } Side effect & $\begin{array}{l}\text { Cyclosporin } \\
(n=14)\end{array}$ & $\begin{array}{l}\text { Placebo } \\
(n=13)\end{array}$ \\
\hline Tremor & 4 & 2 \\
Painful paraesthesiae & 4 & - \\
Hot flushes & 4 & - \\
Headache & 1 & 1 \\
Tiredness & 2 & 2 \\
Epigastric burning & 4 & 2 \\
Nausea & 5 & 1 \\
Loss of appetite & - & 2 \\
Constipation & 1 & 1 \\
Belching & - & 1 \\
Hair growth & 4 & 1 \\
Hair loss & - & 1 \\
Pustules & 2 & - \\
Gum hyperplasia & 3 & - \\
Numb feeling in the lips & 1 & - \\
Dry mouth & 1 & - \\
Increased urinary frequency & 1 & 1 \\
Cystitis & - & - \\
Sinusitis & 1 & - \\
Influenza-like disease & 1 & - \\
Epistaxis & 1 & \\
\hline
\end{tabular}

whereas in four cyclosporin patients the diastolic blood pressure increased $15-20 \mathrm{mmHg}$. This rise in blood pressure was controlled by a reduction of salt intake in three patients and chlorothiazide diuretic therapy in one patient. All these patients also had impaired kidney function.

Five patients of the cyclosporin group and three of the placebo group had a rise in body weight of $5 \mathrm{~kg}$ or more. Three of these patients, all belonging to the cyclosporin group, also had an impaired creatinine clearance.

\section{TOLERABILITY}

Only one patient of the cyclosporin group and five patients of the placebo group did not complain of side effects. A large variety of side effects were reported. They are listed in Table IV. The painful paraesthesias were found to be the most annoying side effect. One patient's denture did not fit any more because of gingival hyperplasia. None of the patients requested a dose reduction.

\section{Discussion}

The results of our study suggest that cyclosporin has a suppressive effect on the course of an ongoing immune response in the form of a severe idiopathic posterior uveitis, panuveitis, or intermediate uveitis, but that this effect is not lasting after dose reduction in which the predose blood concentrations of cyclosporin fall below 200-300 $\mu \mathrm{g} / \mathrm{l}$. The data, however, did not reach statistical significance at the $5 \%$ level. Although it should be noted that our method of using cyclosporin was chosen for the purpose of the investigation, our findings are in accordance with previously published pilot studies. Müftüoglu and associates $^{18}$ treated 11 patients with Behçet's disease with three-month courses of cyclosporin. They observed a rebound phenomenon in all but one patient on the withdrawal of the medication. Similar findings were reported by Graham and associates, ${ }^{19}$ who treated nine patients with severe refractory posterior uveitis. In five patients the uveitis relapsed after dose reduction or withdrawal of cyclosporin. At that moment four patients had been treated for three to six months and one for only three days. All seven patients with Behçet's disease treated by Nussenblatt and associate ${ }^{20}$ required continuation of cyclosporin therapy after six to 21 months.

In evaluating the beneficial effect of cyclosporin in our study we have to take into consideration the possibility that cyclosporin may reduce the elimination of corticosteroids in the liver, resulting in higher blood concentrations and a potentiation of the effects of prednisone. ${ }^{27}$ The pharmacokinetic interactions between corticosteroids and cyclosporin, however, are far from completely characterised, and a recent study by Frey and associates ${ }^{29}$ contradicted these earlier reports.

It is difficult to believe that this possible prednisone potentiating effect is the only mechanism of action of cyclosporin in uveitis. First, the corticosteroid induced lymphocytopenia and eosinopenia did not significantly differ between both treatment groups. And, second, in six of the nine patients considered a treatment failure one to seven months elapsed between discontinuation of prednisone and the relapse of uveitis. It seems unlikely that a reported 2-5-fold increase of the plasma half-life of corticosteroids (that is, 3-7 h) is responsible for this effect in patients receiving only low doses of prednisone.

Cyclosporin is a nephrotoxic drug. ${ }^{30}$ Four patients of the cyclosporin group had a reduction in creatinin clearance exceeding $25 \%$ of the baseline value during the study. None of them had pre-existent hypertension, diabetes mellitus, sarcoidosis, or Behçet's disease, and all had a normal pretreatment creatinin level. In one of them serum creatinin levels still remained approximately $40 \%$ above baseline values four months after cyclosporin was discontinued. On the basis of the findings of Palestine and associates $^{31}$ and Svenson and associates ${ }^{32}$ one can expect morphological damage like an arteriolopathy and a striped form of interstitial fibrosis with tubular atrophy in the kidneys of this patient. It is noteworthy that such a reduction in renal function could occur at rather small cumulative doses - namely, $1311 \mathrm{mg} / \mathrm{kg}$ over a period of five months and with a cyclosporin predose blood level never exceeding $400 \mu \mathrm{g} / \mathrm{l}$. This is the more alarming since cyclosporin does not seem to offer a definite cure for uveitis and in all probability therefore has to be administered for longer periods. Moreover some types of uveitis such as sarcoidosis and Behçet's disease may be accompanied by a reduced kidney function. On the other hand the side effects of corticosteroids may also be serious. Before entering the study one of our patients, for example, had a vertebral fracture with spinal cord compression due to osteoporosis. Transition to cyclosporin therapy can offer the opportunity to recover from the side effects of prolonged systemic corticosteroid therapy without resort to antineoplastic chemotherapy or a reactivation of the intraocular inflammation.

In contrast to Palestine and associates ${ }^{33}$ we failed to detect any relationship between cyclosporin treatment and erythrocyte sedimentation 
rate or haemoglobin concentration, neither in the individual patient nor in the two groups as a whole. None of the patients became anaemic during this study.

Our patients personally tolerated cyclosporin well, and the diverse reported subjective side effects vanished before the intractable uveitis they all had. None of them therefore demanded a dose reduction.

In conclusion, the results of this doublemasked placebo-controlled study indicate that cyclosporin has a place between corticosteroids and cytostatic agents in the treatment of severe chronic idiopathic uveitis.

We are indebted to the outpatient nurses, secretaries, and technologist of the participating ophthalmological departments for their cooperation in this study, to Dr M R Beintema, Dr G Dijkman, Dr R vd Gaag, Dr Z Timmermans, Dr P J Kruit, Professor Dr P T V M de Jong, and Professor Dr G H M van Lith for their constructive criticism, to Mr J C J M Stokvis, Sandoz, for supplying cyclosporin and placebo, and to Ms C H M Muijlwijk, Ms PM L de Zeeuw, and Mr C B Schotel for help in preparing the manuscript.

1 Henderly DE, Genstler AJ, Smith RE, Rao NA. Changing patterns of uveitis. Am F Ophthalmol 1987; 103: 131-6.

2 Canadian Multicentre Transplant Study Group. A randomized clinical trial of cyclosporine in cadaveric renal transplantation. N Engl F Med 1983; 309: 809-15.

3 European Multicentre Trial Group. Cyclosporine in cadaveric renal transplantation; One year follow-up of a multicentre renal transplantation; One year
trial. Lancet 1983; ii: 986-9.

4 Ringdèn $O$. Cyclosporine in allogeneic bone marrow transplantation. Transplantation 1986; 42: 445-52.

5 Deeg HJ, Storb R, Gerhard-Miller L, Shulman HM, Weiden PL, Thomas ED. Cyclosporin A, a powerful immunosuppressant in vivo and in vitro in dogs, fails to induce tolerance. Transplantation 1988; 29; 230-5.

6 Marquet RL, Heystek GA, Cobussen AC, Niessen GJ, Jeekel J. Cyclosporin A prolongs graft survival in presensitized J. Cyclosporin A prolongs graft survival
animals. Transplant Proc 1983; 15: 518-9.

7 Schulak JA, Monson D, Shelby J, Corry RJ. Abrogation of second-set rejection with cyclosporine. Transplantation 1983; 36: 289-93.

8 Pettersson E, Ahonen J, Eklund B, et al, Treatment of acute rejection with cyclosporine. Transplant Proce 1984; 16: 1205-7.

9 Powles RL, Clink H, Sloane J, Barrett AJ, Kay HEM, McElwain TJ. Cyclosporin A for the treatment of graftversus-host disease in man. Lancet 1978; ii: 1327-8.

10 Mochizuki M, Nussenblatt RB, Kuwabara T, Gery I. Effects of cyclosporine and other immunosuppressive drugs on experimental autoimmune uveoretinitis in rats. Invest Ophthalmol Vis Sci 1985; 26: 226-32.
11 Fite KV, Pardue S, Bengston L, Hayden D, Smyth JR. Effects of cyclosporine in spontaneous, posterior uveitis. Curr Eye Res 1986; 5: 787-96.

12 Striph G, Doft B, Rabin B, Johnson B. Retina S antigeninduced uveitis. Arch Ophthalmol 1986; 104: 114-7.

13 Nussenblatt RB, Rodrigues MM, Salinas-Carmona MC, Gery I, Cevario S, Wacker W. Modulation of experimental 1, Cevario S, Wacker W. Modulation of experimental autoimmune uveitis

14 Nussenblatt RB, Palestine AG, Chan CC. Cyclosporin A therapy in the treatment of intraocular inflammatory disease resistant to systemic corticosteroids and cytotoxic agents. Am $\mathcal{F}$ Ophthalmol 1983; 96: 275-82.

15 Nissen C, Bendtzen K, Tvede N, Andersen V. The treatment of presumed non-infective uveitis with cyclosporin A. Acta Ophthalmol (Kbh) 1985; 63 (suppl 173): 72-3.

16 Quentin CD, Vogel M. Ciclosporin-Behandlung bei Uveitis. Klin Monatsbl Augenheilkd 1986; 188: 248-50.

17 Radda TM, Gnad HD. Cyclosporin A in der Augenheilkunde. Radda TM, Gnad HD. Cyclosporin A in der A.
Klin Monatsbl Augenheilkd 1984; 185; 441-3.

18 Müftüoglu AUU, Pazarli H, Yurdakul S, et al. Short term cyclosporin A treatment of Behçet's disease. $\mathrm{Br} \mathcal{F}$ Ophthalmol 1987; 71: 387-90.

19 Graham EM, Sanders MD, James DG, Hamblin A, Kaspgrochowska E, Dumonde D. Cyclosporin A in the treatment of posterior uveitis. Trans Ophthalmol Soc UK 1985; 104: 146-51.

20 Nussenblatt RB, Palestine AG, Chan CC, Mochizuki M, Yancey K. Effectiveness of cyclosporin therapy for Behçet's disease. Arthritis Rheum 1985; 28: 671-9.

21 Ffrench-Constant C, Wolman R, James GD. Cyclosporin in Behçet's disease. Lancet 1983; ii: 454.

22 Lubin JR, Albert DM, Weinstein M. Sixty-five years of sympathetic ophthalmia: a clinicopathologic review of 105 cases (1913-1978). Ophthalmology 1980; 87: 109-21.

23 BenEzra D, Cohen E. Treatment and visual prognosis in Behçet's disease. Br $\mathcal{F}$ Ophthalmol 1986; 70: 589-92.

24 Kimura SK, Thygeson P, Hogan MJ. Signs and symptoms of uveitis. Am f Ophthalmol 1959; 47: 155-70.

25 Johnston A, Marsden JT, Holt DW. The United Kingdom cyclosporin quality assessment scheme. Ther Drug Monit 1986; 8: $200-4$.

26 Cockroft DW, Gault MH. Prediction of creatinine clearance from serum creatinine. Nephron 1976; 16: 31-41.

27 Öst L. Effects of cyclosporin on prednisolone metabolism. Lancet 1984; i: 451 .

28 Langhoff E, Madsen S, Flachs H, Olgaard K, Ladefoged J, Hvidberg EF. Inhibition of prednisolone metabolism by cyclosporine in kidney-transplanted patients. Transplantation 1985; 39: 107-9.

29 Frey FJ, Schnetrer A, Horber FF, Frey BM. Evidence that cyclosporine does not affect the metabolism of prednisolone cyclosporine does

30 Myers BD. Cyclosporine nephrotoxicity. Kidney Int 1986; 30: 964-74.

31 Palestine AG, Austin HA, Balow JE, et al. Renal histopathologic alterations in patients treated with cyclosporine for uveitis. N Engl F Med 1986; 314: 1293-8.

32 Svenson K, Bohman SO, Hällgren R. Renal interstitial fibrosis and vascular changes: occurrence in patients with autoimmune diseases treated with cyclosporine. Arch Intern Med 1986; 146: 2007-10.

33 Palestine AG, Nussenblatt RB, Chan CC. Side effects of systemic cyclosporine in patients not undergoing transplantation. Am $\mathcal{F}$ Med 1984; 77: 652-8. 